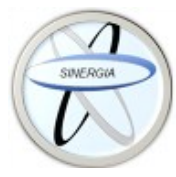

\title{
Depresión postparto
}

\section{Postpartum depression}

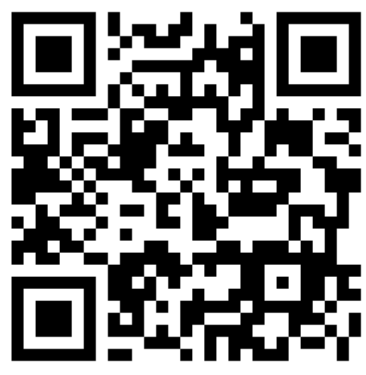

Recibido 17/07/2021
Corregido $20 / 07 / 2021$

${ }^{1}$ Dra. Adriana Acuña Alvarado Hospital Metropolitano, San José, Costa Rica

(i) https://orcid.org/0000-0003-1904-2262

${ }^{2}$ Dra. Estefanía Ramírez Zumbado Hospital San Vicente de Paul, Heredia, Costa Rica

(iD) https://orcid.org/0000-0002-9683-2316

${ }^{3}$ María Fabiola Azofeifa Zumbado Investigadora independiente, Heredia, Costa Rica

(1D) https://orcid.org/0000-0002-9376-299X

\section{RESUMEN}

La depresión postparto es un padecimiento que puede afectar alrededor de $25 \%$ de la población femenina después de un embarazo. Es un trastorno del estado de ánimo que ocurre en los primeros 12 meses después del parto y que se encuentra dentro de un espectro de diagnósticos psiquiátricos relacionados con el embarazo. Las adaptaciones corporales, psicológicas y sociales, tales como el cambio de rol a nivel familiar, el vínculo con él bebé, la nueva responsabilidad asumida y los cambios en el cuerpo pueden alterar el estado de ánimo y producir una depresión posparto. Es necesario el abordaje y detección inmediata de este tipo de padecimiento para tratar de evitar severidad del trastorno que puede llevar a repercusiones tanto en la madre como en él bebe. Este articulo trata de hacer una revisión bibliográfica de la depresión postparto para así conocer más a fondo sobre este padecimiento y que haya una mejor valoración y abordaje de estas pacientes de manera temprana.

PALABRAS CLAVE: postparto; depresión; embarazo; psicológico.

\section{ABSTRACT}

Postpartum depression is a disease that affects around $25 \%$ of the female population after pregnancy. It's a disease associated with behavioral changes in the first 12 months postpartum and is one of the possible behavioral diagnosis related to postpartum. The social, psychological and physical adaptations that women undergo; in addition to the changes in a family, sentimental bondage with the baby, new responsibility as a mother and the physical changes in her body may cause depression. Therefore, early and immediate detection of this type of 
disease is necessary to try and avoid severity that can cause repercussions in the mother as well as the newborn. This article is a bibliographic review that gives an overview of postpartum depression and the specific details for a better evaluation of these patients in early stages.

KEYWORDS: postpartum; depression; pregnancy; behavioral.

${ }^{1}$ Médica general, graduada de la Universidad de Ciencias Médicas (UCIMED). Cód. MED14606. Correo: adriac2192@gmail.com

${ }^{2}$ Médica general, graduada de la Universidad de Ciencias Médicas (UCIMED). Cód. MED14591. Correo: nia erz@me.com

${ }^{3}$ Estudiante de la Ciencias Médicas (UCR). Correo: mafabiola15@gmail.com

\section{INTRODUCCIÓN}

La depresión posparto es una patología frecuente que logra afectar alrededor de $25 \%$ de la población femenina. Las adaptaciones tanto físicas y psicológicas en las madres pueden asociarse a un conflicto de intereses entre sus deseos personales y las nuevas responsabilidades como madre. Esta nueva etapa predispone a un estrés psicológico y etapa de vulnerabilidad para la aparición de un trastorno de estado de ánimo. Para minimizar esta problemática se pueden establecer una serie de medidas con el fin de prevenir la aparición del trastorno. Los cambios recientes en el abordaje y reconocimiento temprano de esta patología nos llevaran a la prevención de cuadros de severidad al igual que una captación e inicio de tratamiento, tanto conservador como farmacológico de manera óptima. Se revisará con detalla esta patologia y así se expondrá su detección, prevención y tratamiento para un manejo precoz.

\section{MÉTODO}

Se realizo una revisión bibliográfica exhaustiva del tema Depresión Posparto a partir de múltiples artículos científicos y libros en los últimos 15 años, publicados en revistas indexadas. Estos nos ayudan a definir y valorar con mayor exactitud este padecimiento. Esto así llevara a un conocimiento más preciso de esta enfermedad para una detección temprana y también un manejo óptimo de ella. Cada uno de estos textos fueron valorados y revisados con detalle para poder lograr un resumen científico y basado en evidencia de esta patología. Los artículos y textos debían cumplir requisitos tales como claridad y definición precisa de depresión posparto, elaboración y desglose de patología y medidas preventivas y tratamiento; todo esto son consideraciones importantes para poder hacer una revisión completa del tema. Con esto se busca una revisión bibliográfica clara y concisa, la cual va a llevar a un mejor conocimiento de ella y detección precoz.

\section{DEFINICIÓN}

La depresión posparto es un trastorno del estado de ánimo, inicia en los primeros 12 meses posterior al parto y presenta síntomas por al menos dos semanas (1). Asimismo, algunos autores indican que puede tener una duración de hasta catorce meses ya que el tiempo que se prolongue la enfermedad depende de la severidad (2). Se considera que esta alteración surge en el periodo puerperio, como respuesta a cambios bioquímicos, hormonales, psicológicos y sociales (3).

Además, algunos autores como Terreno et al (5) indican que entre un 8 y $25 \%$ de las mujeres presentan este trastorno. Mientras 
que Tortaja (4) mencionan que en diferentes países este número es de alrededor del $13 \%$. Sin embargo, otros artículos establecen un intervalo de 10 a 18\% (6). Más recientemente, se ha considerado que este padecimiento afecta entre un 15 y $20 \%$ de las mujeres en esta etapa, lo cual da como resultado un aproximado de 800000 casos anualmente (7). A lo anterior cabe agregar que esta condición es más común en madres adolescentes (26\%) y mujeres de condiciones económicas bajas $(38,2 \%)(14)$. Es importante comprender que además de la depresión posparto, también existen las condiciones de psicosis y tristeza posparto. La tristeza posparto es popularmente conocida como Baby Blues, esta suele aparecer entre los dos y cuatro días posteriores al parto, presenta una duración e intensidad variable y los síntomas son de alivio espontaneo y no requieren atención médica (8). Mientras que, la psicosis posparto consiste en un episodio afectivo con síntomas psicóticos y es más común en madre primerizas (9).

\section{CAUSAS}

Los factores que originan la depresión posparto pueden ser de tipo bioquímicos, hormonales, psicológicos y sociales. Loaiza y colaboradores (11) sugieren que dentro de las variables bioquímicas destaca la disminución abrupta en los niveles de esteroides gonadales. Sin embargo, indican que esta condición, por sí sola, no desencadena la enfermedad, debido a que el padecimiento está influenciado por cambios hormonales y deficiencias sociales. Estas deficiencias sociales corresponden a la carencia de redes de apoyo, principalmente en madres adolescentes, embarazos no deseados y condiciones económicas bajas (1). Por otra parte, las adaptaciones corporales, psicológicas y sociales, tales como el cambio de rol a nivel familiar, el vínculo con él bebé, la nueva responsabilidad asumida y los cambios en el cuerpo pueden alterar el estado de ánimo y producir una depresión posparto $(3,8)$. Además, Decherney et al (10) mencionan que el riesgo principal es un cuadro de depresión previo al embarazo, también este factor suele producir síntomas y consecuencias más graves.

\section{DIAGNOSTICO}

El diagnóstico de esta condición, se deben tomar en cuenta tanto elementos conductuales como orgánicos. Dentro de los conductuales los más comunes son humor depresivo, falta de interés o placer en actividades, alteración del sueño, pérdida de energía, agitación o retraso psicomotor, sentimientos excesivos de culpa, disminución de la concentración e ideas suicidas (7). En relación con los factores de tipo orgánico, se puede mencionar el hipotiroidismo y variaciones en el metabolismo de hormonas tiroideas, así como, cambios en funciones neurotransmisoras y disminución de algunas citoquinas inflamatorias (12).

Por otra parte, al ser la depresión posparto un problema de salud pública y dadas las repercusiones en el bienestar de la madre y del bebé es importante realizar un diagnóstico temprano y oportuno (14). Sin embargo, existe una percepción errónea de que este trastorno está asociado con debilidad y una inadecuada maternidad, por lo cual muchas mujeres reprimen los sentimientos de tristeza para no ser juzgadas (15). A pesar de que la condición se puede desarrollar en los primeros 12 meses posterior al parto, generalmente, los síntomas empiezan a aparecer en las 
primeras dos semanas después de que él bebé nace (14).

No obstante, Meléndez et al (16) afirma que se puede manifestar durante el periparto hasta las cuatro semanas posparto, dentro de esta etapa los síntomas más frecuentes son la tristeza, el llanto, poca expresividad y rechazo al recién nacido. Por lo tanto, para lograr una detección temprana, es necesario realizar un examen entre el tercer y sexto día poshospitalario (7). Además, según Seras et al (17) la depresión posparto es un padecimiento frecuente pero insuficientemente detectada.

Debido a lo anterior, es prioritario abordar temas que planteen medidas para detectar, atender y comprender este trastorno. Para realizar un adecuado diagnóstico González y colaboradores (3) plantean una serie de instrumentos, entre los cuales se encuentran la Entrevista Clínica Estructurada, la Escala de Depresión Posparto, la Escala de Depresión del Centro de Estudios Epidemiológicos, el Cuestionario de Depresión de Beck II y el Cuestionario sobre la Salud del Paciente. Sin embargo, en vista de su rapidez y facilidad tanto de aplicación como de compresión, actualmente la más empleada es la Escala de Depresión Posnatal de Edimburgo (18).

De esta manera, la Escala de Depresión Posnatal de Edimburgo consiste en 10 preguntas relacionadas con disforia, ansiedad, sentimientos de culpa, ideas suicidas y concentración (18). Por otra parte, Solano et al (7) indican que el proceso de valoración de depresión posparto es similar al proceso de valoración de depresión en pacientes que no estén en este periodo. Dicha evaluación, trata asuntos de historial médico psiquiátrico y general, examen físico y estado mental; además, incluye pruebas de laboratorio para descartar una afección médica general que presente síntomas similares (19).

Además de la identificación de comportamientos negativos, para realizar el diagnóstico también es necesario prestar atención a conductas positivas. Estas conductas se consideran como factores protectores, entre los cuales están: estilos cognitivos positivos, niveles elevados de autoestima maternal, buenas relaciones de pareja, percepción materna de apoyo y alimentación del bebé con lactancia maternal (3). En este punto, para desarrollar un diagnóstico adecuado, también es importante conocer aspectos del entorno social y familiar de la madre (14).

\section{TRATAMIENTO}

A partir de los resultados obtenidos, en caso de que la madre padezca de depresión posparto se toman diversas medidas según la severidad del caso. Si presenta síntomas leves, normalmente, son referidas al departamento de urgencias en donde se le asigna una consulta psiquiátrica para una mayor evaluación (7).

Mientras que, si se trata de un caso severo se recurre a la hospitalización del paciente para así disminuir el riesgo de suicidio (20). Por otro lado, la depresión posparto posee dos diagnósticos diferenciales: depresión posparto leve-moderada y depresión posparto severa (7). En el primer caso, los pacientes no presentan ideas suicidas, deterioro del funcionamiento $y$ complicaciones psicóticas (21). Algunos de los tratamientos para este diagnóstico son la terapia cognitivo-conductual, psicoterapia interpersonal, asesoramiento no directivo y psicoterapia psicodinámica.

En relación con la depresión posparto severa, los pacientes a menudo presentan pensamientos suicidas, deterioro grave del 
funcionamiento y mal juicio, los factores anteriores están asociados a un riesgo de daño inminente (7). Para la atención de estos casos se recomienda el uso de fármacos antidepresivos (22). No obstante, antes de recetar estas sustancias debe analizarse cada paciente individualmente, debido a que en ocasiones se han detectado riesgos para el lactante (14). Por otra parte, González et al (3) considera que el tratamiento debe estar consolidado tanto por terapia psicológica como por farmacología.

\section{PREVENCIÓN}

Para minimizar esta problemática se pueden establecer una serie de medidas con el fin de prevenir la aparición del trastorno. Entre las prácticas que se pueden llevar a cabo está apreciar la maternidad, así como reconocer el valor que la madre sigue teniendo como ser humano (23). Además, se puede recurrir a la implementación de programas que brinden apoyo a las madres después del parto, promover el empoderamiento social de la mujer y actividades que fortalezcan el vínculo entre la madre y él bebé (24).

Por otra parte, la sociedad también juega un rol importante en la forma en la que se maneja la gestación y los efectos de esta. De manera que, es importante consolidar redes de apoyo, así como replantearse las presiones de perfección asociadas a la maternidad, ya que estos elementos funcionan como estresores adicionales y pueden ser un limitante para buscar ayuda (14). Lo anterior dificulta alcanzar un tratamiento efectivo, lo que perturba el bienestar de la mamá, él bebé y el núcleo familiar (8). Con base en lo anterior, se resalta la importancia de considerar las implicaciones de la depresión posparto para los hombres padres, otros hijos y él bebé, ya que son los de mayor proximidad a la mujer (25).

\section{CONCLUSIONES}

La depresión posparto es un padecimiento frecuente; por lo cual debe de ser parte de las evaluaciones iniciales impartidas después del embarazo. Este padecimiento es causado por múltiples factores tanto ambientales como biológicos en la madre. Es por esto que se requiere de una valoración temprana para así poder brindarle a las pacientes un tratamiento adecuado y oportuno en el momento de detección. La detección temprano llevara a la prevención de cuadros severos que pueden afectar tanto a la madre como al recién nacido.

\section{Los autores declaran no tener conflicto de interés.}

\section{REFERENCIAS}

1. Escobar, J., Pacora, P., Custodio, N., y Villar, W. (2009, June). Depresión posparto: ¿se encuentra asociada a la violencia basada en género? In Anales de la Facultad de Medicina (Vol. 70, No. 2, pp. 115-118). UNMSM. Facultad de Medicina.

2. Jiménez Serrano, S. (2014). Desarrollo de modelos predictivos y una aplicación móvil para la predicción de la depresión posparto.

3. González, R. A., Tello, V. L., Salán, M. M., Fernández, P. F., del Rivero, V. D. C. A., y Díaz, M. N. F. (2017). Actualización en depresión posparto. TIEMPOS DE ENFERMERÍA Y SALUD, 1(3), 18-22.

4. Tortajada, S., Garcia-Gomez, J. M., Vicente, J., Sanjuán, J., de Frutos, R., Martín-Santos, R., ... y Robles, M. (2009). Prediction of postpartum depression using multilayer perceptrons and pruning. Methods of information in medicine, 48(03), 291-298.

5. Terrén, C. A., Esteve, L. G., Navarro, P., Aguado, J., Ojuel, J., y Tarragona, M. J. (2003). Prevalencia de la depresión posparto en las madres españolas: comparación de la estimación mediante la entrevista clínica estructurada y la escala de depresión posparto de Edimburgo. Medicina clínica, 120(9), 326-329. 
6. Guintivano, J., Arad, M., Gould, T. D., Payne, J. L., y Kaminsky, Z. A. (2014). Antenatal prediction of postpartum depression with blood DNA methylation biomarkers. Molecular psychiatry, 19(5), 560-567.

7. Solano, M. S., Mejía, A. L. P., y Madrigal, J. C. (2019). Abordaje clínico y manejo de la depresión posparto. Revista Médica Sinergia, 4(6), 90-99.

8. Medina-Serdán, E. (2013). Diferencias entre la depresión posparto, la psicosis y la tristeza posparto. Perinatología y reproducción humana, 27(3), 185-193.

9. Heron J, McGuinness M, Robertson E, Craddock $\mathrm{N}$ y Jones, I. 2008. Early postpartum symptoms in puerperal psychosis. BJOG, 115, 348-53.

10. Decherney, A., Laufer, N., Nathan, L. y Roman, A. (2014). Diagnóstico y tratamiento ginecoobstétricos. XI Edición. Nueva York: Lange Medical Book/McGraw-Hill.

11. Loaiza, N. V., Dachner, A. P., y Morales, J. A. V. (2019). Generalidades y diagnóstico de la depresión posparto. Revista Médica Sinergia, 4(7), e261-e261.

12. Bourget, D., Grace, J., y Whitehurst, L. (2007). A review of maternal and paternal filicide. JournalAmerican Academy of Psychiatry and The Law, 35(1), 74.

13. Shariatpanaahi, M. V., Shariatpanaahi, Z. V., Moshtaaghi, M., Shahbaazi, S. H., y Abadi, A. (2007). The relationship between depression and serum ferritin level. European journal of clinical nutrition, 61(4), 532-535.

14. Mendoza, C., y Saldivia, S. (2015). Actualización en depresión posparto: el desafío permanente de optimizar su detección y abordaje. Revista médica de Chile, 143(7), 887-894.

15. Malpartida, A. M. K. (2020). Depresión posparto en atención primaria. Revista Médica Sinergia, 5(02), 355-355.

16. Meléndez, M., Díaz, M., Bohorjas, L., Cabaña, A., Casas, J., Castrillo, M., y Corbino, J. (2017). Depresión posparto y los factores de riesgo. Salud, 21(3), 7-12.

17. Lasheras, G., Sender, B. F., y Serra, B. (2014). Detección in situ de la depresión posparto (DPP) y alteración del vínculo materno-filial en el Hospital Universitario Quirón Dexeus. Resultados preliminares. Cuadernos de medicina psicosomática y psiquiatría de enlace, (109), 2231.

18. Dois, C. A. 2012. Actualizaciones en depresión posparto. Rev Cub Obst y Ginecol, 38(4), 576586.
19. Sines, E., Syed, U., Wall, S., y Worley, H. (2007). Postnatal care: A critical opportunity to save mothers and newborns. Policy Perspectives on Newborn Health, 1(7).

20. Yonkers, K. A., Vigod, S., y Ross, L. E. (2012). Diagnosis, pathophysiology, and management of mood disorders in pregnant and postpartum women. Focus, 10(1), 51-66.

21. Putnam K, Robertson-Blackmore E, Sharkey K, Payne J, Bergink V., ... y Munk-Olsen T. 2015. Heterogeneity of postpartum depression: a latent class analysis. Lancet Psychiatry, 2(1). 67. https://doi.org/10.1016/S2215-0366(14)00055-8

22. Meltzer-Brody, S., y Jones, I. (2015). Optimizing the treatment of mood disorders in the perinatal period. Dialogues in clinical neuroscience, 17(2), 207.

23. Nóblega, M. 2009. La maternidad en la vida de las adolescentes: implicancias para la acción. Lima, Rev Psicol, 27(1), 29-54.

24. Masías, S. M. A., y Arias, G. W. L. (2018). Prevalencia de la depresión post parto en Arequipa, Perú. Revista Medica Herediana, 29(4), 226-231.

25. Barbosa, M. A. R. D. S., y Ângelo, M. (2016). Experiencias y significados de la depresión posparto de mujeres en el contexto de la familia. Enfermería Global, 15(42), 232-255. 